\title{
The projection operator in a Hilbert space and its directional derivative. Consequences for the theory of projected dynamical systems
}

\author{
George Isac and Monica G. Cojocaru \\ (Communicated by Lars-Erik Persson)
}

2000 Mathematics Subject Classification. 37C 75

Keywords and phrases. Directional derivative of projection operators, projected dynamical systems, Hilbert space.

\footnotetext{
Abstract. In the first part of this paper we present a representation theorem for the directional derivative of the metric projection operator in an arbitrary Hilbert space. As a consequence of the representation theorem, we present in the second part the development of the theory of projected dynamical systems in infinite dimensional Hilbert space. We show that this development is possible if we use the viable solutions of differential inclusions. We use also pseudomonotone operators.
}

\section{Introduction}

Presently, several authors are interested in the study of variational inequalities and of complementarity problems, from the point of view of dynamical systems. See [10], [12], [31]-[36], [45] among others.

This interest is justified by the many applications of the variational inequalities and complementarity problems to Economics and Engineering. It is well known that variational inequalities and complementarity problems are mathematical tools used in the study of equilibrium as it is considered 
in Economics or the equilibrium considered in Physics and in Engineering [2], [7], [13], [22]-[25]. The study of perturbed equilibrium states is therefore important in many practical problems.

The study, from the point of view of dynamical systems of variational inequalities and of complementarity problems, is now done via a projected dynamical system. [10], [12], [31]-[36], [45].

It is known that the critical points of the projected dynamical system associated to a variational inequality (resp. to a complementarity problem) are exactly the solutions of the variational inequality (resp. of the complementarity problem).

In Nagurney and Zhang's book [36], several stability results for solutions of projected dynamical systems associated to a variational inequality, are obtained using Dupuis' representation theorem proved by P. Dupuis in $\boldsymbol{R}^{n}$ [11]. Dupuis' result is a representation theorem for the directional derivative of the projection operator.

For many equilibrium problems in Elasticity, Fluid Mechanics and Engineering it is necessary to develop the theory, of projected dynamical systems, in an infinite dimensional Hilbert spaces. In many practical problems we have functional Hilbert spaces. To do this, a natural question is to know if Dupuis' representation theorem is valid in an infinite dimensional Hilbert space and if yes, we must know its form.

In this paper, we show that in infinite dimensional Hilbert spaces, Dupuis' representation theorem is not valid, under the form known in $\boldsymbol{R}^{n}$. However, some parts of this representation result are valid, but with some small modifications. So, we give the form of Dupuis' theorem in an arbitrary infinite dimensional Hilbert spaces. Because of this fact, we note that in infinite Hilbert spaces, the projected dynamical systems must be studied using a natural differential inclusion associated to the projected differential equation. This is the "evolution differential inclusion". We show that in this way, we can study the existence of trajectories and their stability, near a critical point. A consequence of this paper is the fact that now we can develop the theory of projected dynamical systems in infinite dimensional Hilbert spaces. The first part of this development was recently presented in the Ph.D Thesis [3].

\section{Preliminaries}

We will denote by $(H,\langle\cdot, \cdot\rangle)$ an arbitrary Hilbert space. Let $D \subset H$ be a closed convex set. It is known that for any $x \in H$, the projection of $x$ onto $D$, denoted by $P_{D}(x)$ is well defined. We have that $P_{D}(x)$ is the unique 
element in $D$ such that

$$
\left\|x-P_{D}(x)\right\| \leq\|x-y\|, \text { for all } y \in D .
$$

The element $P_{D}(x)$ is also characterized by the following variational inequality

$$
\left\langle P_{D}(x)-x, P_{D}(x)-y\right\rangle \leq 0, \quad \text { for all } y \in D .
$$

Given a mapping $f: H \rightarrow H$, the variational inequality defined by $f$ and $D$ is

$$
V I(f, D):\left\{\begin{array}{l}
\text { find } x_{*} \in D \text { such that } \\
\left.f\left(x_{*}\right), y-x_{*}\right\rangle \geq 0, \text { for all } y \in D .
\end{array}\right.
$$

If $D=\boldsymbol{K}$, where $\boldsymbol{K} \subset H$ is a closed pointed convex cone, then the problem $V I(f, \boldsymbol{K})$ is the complementarity problem defined by $f$ and $\boldsymbol{K}$, i.e.,

$$
C P(f, K):\left\{\begin{array}{l}
\text { find } x_{*} \in K \text { such that } \\
f\left(x_{*}\right) \in K^{*} \text { and } \\
\left\langle x_{*}, f\left(x_{*}\right)\right\rangle=0,
\end{array}\right.
$$

where $\boldsymbol{K}^{*}$ is the dual of $\boldsymbol{K}$, i.e.,

$$
K^{*}=\{y \in H \mid\langle y, x\rangle \geqslant 0, \text { for all } x \in K\} .
$$

If $A \subset H$ is an arbitrary subset, we will denote by $\bar{A}^{\sigma}$ the closure of $A$, with respect to the weak-topology and by $\partial A$ the boundary of $A$ with respect to the norm-topology. We will denote by $A^{0}$ the polar of $A$ i.e.,

$$
A^{0}=\{y \in H \mid\langle y, x\rangle \leq 0, \text { for all } x \in A\},
$$

and by $\operatorname{int} A$, the interior of $A$ with respect to the norm-topology. If $x \in D$, we recall that the closed convex cone

$$
N_{D}(x)=\{\xi \in H \mid\langle\xi, y-x\rangle \leq 0, \text { for all } y \in D\}
$$

is called the normal cone of $D$ at the point $x \in D$. If $S=\{x \in H \mid\|x\|=1\}$, we define $n_{D}(x)=S \cap N_{D}(x)$. The tangent cone of $D$ at the point $x \in D$, is by definition

$$
T_{D}(x)=\overline{\bigcup_{t>0} \frac{1}{t}(D-x)} .
$$

It is known that $N_{D}(x)=\left(T_{D}(x)\right)^{0}$. 
Finally, if $\delta_{0}>0$ is a real number we denote by $B\left(x, \delta_{0}\right)=\{y \in$ $\left.H \mid\|x-y\|<\delta_{0}\right\}$.

The directional derivative of the projection operator $P_{D}$ was studied in [38], [39], [40] and initially in [44]. In [40] it is proved that the directional derivative of operator $P_{D}$ at any point $x \in D$ and along any direction $v$ exists, i.e., $\lim _{\delta \rightarrow 0^{+}} \frac{P_{D}(x+\delta v)-x}{\delta}$, exists if $x \in D$ and $v \in H$ is any arbitrary element (direction).

We denote by $\Pi_{D}(x ; v)=\lim _{\delta \rightarrow 0^{+}} \frac{P_{D}(x+\delta v)-x}{\delta}$. Moreover, in [39] it is proved that $\Pi_{D}(x ; v)=P_{T_{D}(x)}(v)$.

The following results are useful in proving the main result of this paper.

Lemma 1. If $x \in H$ then $P_{D}(x)=y, y \in D$ if and only if $x-P_{D}(x)=$ $\alpha \xi$, where $\alpha \geq 0$ and $\xi \in n_{D}(y)$.

Proof. Indeed, $P_{D}(x)$ is characterized by (1), that is,

$$
\left\langle P_{D}(x)-x, P_{D}(x)-u\right\rangle \leq 0, \text { for all } u \in D,
$$

which implies

$$
\left\langle x-P_{D}(x), u-P_{D}(x)\right\rangle \leq 0, \text { for all } u \in D .
$$

From (3) we deduce that $x-P_{D}(x) \in N_{D}\left(P_{D}(x)\right)=N_{D}(y)$, and therefore there exists $\alpha \geq 0$ and $\xi \in n_{D}(y)$ such that $x-P_{D}(x)=\alpha \xi$.

Corollary. For any $x \in H$, we have $x-P_{D}(x)=\left\langle x-P_{D}(x), n\right\rangle n$, where $\left\langle x-P_{D}(x), n\right\rangle \geq 0$ and $n \in n_{D}\left(P_{D}(x)\right)$.

Lemma 2. In an arbitrary Hilbert space $(H,\langle\cdot, \cdot\rangle)$, if a sequence $\left\{x_{n}\right\}_{n \in N}$ is norm-convergent to an element $x_{*}$ and a sequence $\left\{y_{n}\right\}_{n \in N}$ is weakly convergent to an element $y_{*}$, then $\lim _{n \rightarrow \infty}\left\langle x_{n}, y_{n}\right\rangle=\left\langle x_{*}, y_{*}\right\rangle$.

Proof. For a proof of this result, the reader is referred to [23].

Theorem 3 ([Eberlein-Šmulian]). A set $M$ in a Banach space $(E,\|\cdot\|)$ is relatively weakly compact, if and only if, every sequence $\left\{x_{n}\right\}_{n \in N}$ in $M$ has a weakly convergent subsequence.

Proof. For a proof of this classical result, the reader is referred to [43].

\section{The main result}

In the case when $(H,\langle\cdot, \cdot\rangle)$ is the Euclidean space $\left.\boldsymbol{R}^{n},\langle\cdot, \cdot\rangle\right)$, in 1987, P. Dupuis proved the following result.

Theorem 4 (Dupuis, [11]). If $x \in D$ and $v \in H$, then 
(i) $\Pi_{D}(x, v)=v$, if $x \in$ intD,

(ii) $\Pi_{D}(x, v)=v$, if $x \in \partial D$ and $\sup _{n \in n_{D}(x)}\langle v, n\rangle<0$,

(iii) $\Pi_{D}(x, v)=v-\left\langle v, n^{*}\right\rangle n^{*}$ if $x \in \partial D$ and $\sup _{n \in n_{D}(x)}\langle v, n\rangle \geq 0$ and $n^{*}$ achieves the maximum.

In this section we will show that Theorem 4 is not valid, in the form presented above, in an arbitrary infinite dimensional Hilbert space. However, some parts of this Theorem are valid, but with some modifications. The modifications are necessary since the set $n_{D}(x)$ must be replaced by the set $\bar{S}^{\sigma} \cap N_{D}(x)$, where $\bar{S}^{\sigma}$ is the closure of the set $S=\{x \in H \mid\|x\|=1\}$, with respect to the weak topology. We will give the form of Theorem 4 in an arbitrary Hilbert space.

Theorem 5. Let $(H,\langle\cdot, \cdot\rangle)$ be an arbitrary Hilbert space and $D \subset H a$ non-empty closed convex set.

If $\Pi_{D}(x ; v)=\lim _{\delta \rightarrow 0_{+}} \frac{P_{D}(x+\delta v)-x}{\delta}$, where $x \in D$ and $v \in H$, then we have :

(I) $\Pi_{D}(x ; v)=v$, if $x \in \operatorname{int} D$,

(II) $\Pi_{D}(x ; v)=v$, if $x \in \partial D$, and $\sup _{n \in \bar{S}^{\sigma} \cap N_{D}(x)}\langle v, n\rangle<0$ only if dim $H<\infty$. If $H$ is infinite dimensional this representation is not valid.

(III) $\Pi_{D}(x ; v)=v-\left\langle v, n^{*}\right\rangle n^{*}$, if $x \in \partial D$ and $\sup _{n \in \bar{S}^{\sigma} \cap N_{D}(x)}\langle v, n\rangle>0$, where $n^{*}$ achieves the $\sup _{n \in \bar{S}^{\sigma} \cap N_{D}(x)}\langle v, n\rangle$. Moreover $n^{*}$ is unique.

(IV) $\Pi_{D}(x ; v)=v$, if $x \in \partial D, \sup _{n \in \bar{S}^{\sigma} \cap N_{D}(x)}\langle v, n\rangle=0$, and $\sup _{n \in \bar{S}^{\sigma} \cap N_{D}(x)}\langle v, n\rangle$ has at least a maximizer $n^{0} \neq 0$,

(V) $\Pi_{D}(x ; v)=v-\xi$, where $\xi$ is an undetermined element in $N_{D}(x)$, if $x \in \partial D$, and we are in any case not considered in conclusions $I-I V$.

Proof. I. If int $D \neq \phi$ and $x \in$ int $D$, then there exists $\delta_{0}>0$ such that $B\left(x, \delta_{0}\right) \subset D$. If $v=0$, obviously, in this case $\Pi_{D}(x ; v)=0(=v)$.

Suppose $v \neq 0$. For any $\delta$ such that $0<\delta<\frac{\delta_{0}}{\|v\|}$, we have that $x+\delta v \in B\left(x, \delta_{0}\right)$ and finally we can show that $\Pi_{D}(x ; v)=v$.

Before proving conclusion (II), we remark that, since the set $\bar{S}^{\sigma} \cap N_{D}(x)$ is weakly compact and the linear functional $u \rightarrow\langle u, v\rangle, u \in H$, is weakly continuous, then, $\sup _{n \in \bar{S}^{\sigma} \cap N_{D}(x)}\langle n, v\rangle$ is a finite real number, which is achieved. 
II. If $x \in \partial D$ and $\operatorname{dim} H<\infty$, then in this case, by the proof of the section (ii) of Theorem 4 given in [11] we have that $\Pi_{D}(x ; v)=v$. (We used also the fact that when $\operatorname{dim} H<\infty$ we have that $\left.\bar{S}^{\sigma} \cap N_{D}(x)=n_{D}(x)\right)$.

Now, we suppose that $\operatorname{dim} H=\infty$. In this case the inequality sup $\langle v, n\rangle<0$ is not possible, since $n=0 \in \bar{S}^{\sigma} \cap N_{D}(x)$. Indeed, let $\left\{u_{n}\right\}_{n \in N}$ be an orthonormal sequence in $H$. (This is possible since $\operatorname{dim} H=\infty$ ). Then, for any $\xi \in H$ we have $\sum_{n=1}^{\infty}\left|\left\langle\xi, u_{n}\right\rangle\right|^{2} \leq\|\xi\|^{2}$. (This is the classical Bessel inequality). The last inequality implies that the sequence $\left\{\left\langle\xi, u_{n}\right\rangle\right\}_{n \in N}$ is convergent to zero for any $\xi \in H$. Therefore the sequence $\left\{u_{n}\right\}_{n \in N} \subset S$, is weakly convergent to zero and hence $0 \in \bar{S}^{\sigma} \cap N_{D}(x)$.

III. We suppose $x \in \partial D$ and $\sup _{n \in \bar{S} \cap N_{D}(x)}\langle v, n\rangle>0$.

Because the supremum is achieved, there exists $n^{0} \in \bar{S}^{\sigma} \cap N_{D}(x), n^{0} \neq 0$ such that $\left\langle n^{0}, v\right\rangle>0$ (obviously, $v \neq 0$ ). In this case $P_{D}(x+\delta v) \in \partial D$ for all $\delta \geq 0$. According to Lemma 1 (Corollary), for any $\delta>0$ there is $n \in n_{D}\left(P_{D}(x+\delta v)\right)$ such that

$$
P_{D}(x+\delta v)=x+\delta v-\left\langle x+\delta v-P_{D}(x+\delta v), n\right\rangle n,
$$

where $\left\langle x+\delta v-P_{D}(x+\delta v), n\right\rangle$ must be non negative.

By using (4), we define $\delta_{i} \downarrow 0$ and a sequence $\left\{n_{i}\right\}_{i \in N}$, where $n_{i}$ is corresponding to $\delta_{i}$ and $n_{i} \in n_{D}\left(P_{D}\left(x+\delta_{i} v\right)\right)$. We have that $\left\{n_{i}\right\}_{i \in N}$ is weakly convergent to an element is weakly convergent to an element $n^{*} \in$ $\bar{S}^{\sigma} \cap N_{D}(x)$. Indeed, since $n_{i} \in \bar{S}^{\sigma}$ for any $i \in N$ and $\bar{S}^{\sigma}$ is weakly compact, we have that $\left\{n_{i}\right\}_{i \in N}$ has, by Eberlein-Smulian Theorem (Theorem 3), a weakly convergent subsequence, denoted again by $\left\{n_{i}\right\}_{i \in N}$. Hence, we can suppose that there exists $n^{*}=w-\lim _{i \rightarrow \infty} n_{i}$ and $n^{*} \in \bar{S}^{\sigma}$. Moreover, we have also that $n^{*} \in N_{D}(x)$. Indeed, since $n_{i} \in n_{D}\left(P_{D}\left(x+\delta_{i} v\right)\right)$, we deduce that for any $x^{\prime} \in D$ we have $\left\langle n_{i}, P_{D}\left(x+\delta_{i} v\right)-x^{\prime}\right\rangle \geq 0$.

Because $P_{D}\left(x+\delta_{i} v\right) \stackrel{\|\cdot\|}{\longrightarrow} P_{D}(x)$ and $\left\{n_{i}\right\} \stackrel{(w)}{\longrightarrow} n^{*}$, applying Lemma 2, we deduce that $\left\langle n^{*}, x-x^{\prime}\right\rangle \geq 0$ for all $x^{\prime} \in D$, which implies that $n^{*} \in N_{D}(x)$. Therefore we have $n^{*} \in \bar{S}^{\sigma} \cap N_{D}(x)$.

Using (4) again we deduce the following formula

$$
\frac{P_{D}\left(x+\delta_{i} v\right)-x}{\delta_{i}}=v-\left\langle v, n_{i}\right\rangle n_{i}-\frac{1}{\delta_{i}}\left\langle x-P_{D}\left(x+\delta_{i} v\right), n_{i}\right\rangle n_{i} .
$$


From (5) we obtain that

$$
\begin{cases}\left\langle\frac{P_{D}\left(x+\delta_{i} v\right)-x}{\delta_{i}}, \varphi\right\rangle=\langle v, \varphi\rangle-\left\langle v, n_{i}\right\rangle\left\langle n_{i}, \varphi\right\rangle & \\ -\frac{1}{\delta_{i}}\left\langle x-P_{D}\left(x+\delta_{i} v\right), n_{i}\right\rangle\left\langle n_{i}, \varphi\right\rangle, & \text { for all } \varphi \in H\end{cases}
$$

We denote by $B_{i}=-\frac{1}{\delta_{i}}\left\langle x-P_{D}\left(x+\delta_{i} v\right), n_{i}\right\rangle\left\langle n_{i}\right.$, varphi $\rangle$ and we will show that $\lim _{i \rightarrow \infty} B_{i}=0$. For this, we show first that,

$$
\lim _{i \rightarrow \infty} \frac{1}{\delta_{i}}\left\langle x-P_{D}\left(x+\delta_{i} v\right), n_{i}\right\rangle=0 .
$$

Indeed, because $n_{i} \in n_{D}\left(P_{D}\left(x+\delta_{i} v\right)\right)$, we have

$$
\frac{1}{\delta_{i}}\left\langle x-P_{D}\left(x+\delta_{i} v\right), n_{i}\right\rangle \leq 0
$$

Applying Lemma 2 we have $\lim _{i \rightarrow \infty} \frac{1}{\delta_{i}}\left\langle x-P_{D}\left(x+\delta_{i} v\right), n_{i}\right\rangle=\left\langle-\Pi_{D}(x ; v), n^{*}\right\rangle$ and hence

$$
\left\langle-\Pi_{D}(x ; v), n^{*}\right\rangle \leq 0
$$

Since $n^{*} \in N_{D}(x)$ we obtain $\left\langle x-P_{D}\left(x+\delta_{i} v\right), n^{*}\right\rangle \geq 0$, which implies $\frac{1}{\delta_{i}}\left\langle x-P_{D}\left(x+\delta_{i} v\right), n^{*}\right\rangle \geq 0$ and computing the limit we obtain

$$
\left\langle-\Pi_{D}(x ; v), n^{*}\right\rangle \geq 0
$$

From (8) and (9) we have $\left\langle-\Pi_{D}(x ; v), n^{*}\right\rangle=0$, that is, $(7)$ is true.

Because the sequence $\left\{n_{i}\right\}_{i \in N}$ is weakly convergent, we have that the sequence $\left\{\left\langle n_{i}, \varphi\right\rangle\right\}_{i \in N}$ is bounded for any $\varphi \in H$. Therefore, considering (7) we obtain that $\lim _{i \rightarrow \infty} B_{i}=0$. Computing the limit in (6) we obtain

$$
\left\langle\Pi_{D}(x ; v), \varphi\right\rangle=\langle v, \varphi\rangle-\left\langle v, n^{*}\right\rangle\left\langle n^{*}, \varphi\right\rangle,
$$

or

$$
\left\langle\Pi_{D}(x ; v)-v+\left\langle v, n^{*}\right\rangle n^{*}, \varphi\right\rangle=0, \text { for all } \varphi \in H .
$$

which implies that

$$
\Pi_{D}(x ; v)=v-\left\langle v, n^{*}\right\rangle n^{*} .
$$


We must show that $n^{*}$ is a maximizer in $\sup _{n \in \bar{S}^{\sigma} \cap N_{D}(x)}\langle v, n\rangle$.

Indeed, because $P_{D}\left(x+\delta_{i} v\right) \in \partial D \subseteq D$, we have

$$
\left\langle P_{D}\left(x+\delta_{i} v\right)-x, n\right\rangle \leq 0, \text { for all } n \in \bar{S}^{\sigma}, \cap N_{D}(x),
$$

which implies

$$
\sup _{n \in \bar{S}^{\sigma} \cap N_{D}(x)}\left\langle P_{D}\left(x+\delta_{i} v\right)-x, n\right\rangle \leq 0
$$

and using formula (5) we deduce

$$
\sup _{n \in \bar{S}^{\sigma} \cap N_{D}(x)}\left\langle\delta_{i}\left[v-\left\langle v, n_{i}\right\rangle n_{i}-\frac{1}{\delta_{i}}\left\langle x-P_{D}\left(x+\delta_{i} v\right), n_{i}\right\rangle n_{i}\right], n\right\rangle \leq 0
$$

which implies (since $\delta_{i}>0$ for all $i \in N$ ),

$$
\sup _{n \in \bar{S}^{\sigma} \cap N_{D}(x)}\left\langle v-\left\langle v, n_{i}\right\rangle n_{i}-\frac{1}{\delta_{i}}\left\langle x-P_{D}\left(x+\delta_{i} v\right), n_{i}\right\rangle n_{i}, n\right\rangle \leq 0
$$

or

$$
\sup _{n \in \bar{S}^{\sigma} \cap N_{D}(x)}\left[\left\langle v-\left\langle v, n_{i}\right\rangle n_{i}, n\right\rangle-\frac{1}{\delta_{i}}\left\langle x-P_{D}\left(x+\delta_{i} v\right), n_{i}\right\rangle\left\langle n_{i}, n\right\rangle\right] \leq 0
$$

Computing the limit we obtain

$$
\sup _{n \in \bar{S}^{\sigma} \cap N_{D}(x)}\left[\langle v, n\rangle-\left\langle v, n^{*}\right\rangle\left\langle n^{*}, v\right\rangle\right] \leq 0 .
$$

We remember that we are in the case

$$
\sup _{n \in \bar{S}^{\sigma} \cap N_{D}(x)}\langle v, n\rangle>0 .
$$

We have that (11) and (12) imply $n^{*} \neq 0$.

Indeed, if $n^{*}=0$, then $\left\langle v, n^{*}\right\rangle\left\langle n^{*}, n\right\rangle=0$, implies $\sup _{n \in \bar{S}^{\sigma} \cap N_{D}(x)}\langle v, n\rangle \leq 0$, which is a contradiction of (12). Hence, in (11) we must have $n^{*} \neq 0$. Now we show that $\left\langle v, n^{*}\right\rangle \geq 0$.

Indeed, if $\left\langle v, n^{*}\right\rangle<0$, then in this case, as in the proof of conclusion (II) we can show that there exists $\delta_{*}>0$ such that $x+\delta_{*} v \in D$.

Indeed, if we suppose that $x+\delta v \notin D$ for all $\delta>0$, then using the sequence $\left\{n_{i}\right\}_{i \in N}$ which is weakly convergent to $n^{*}$, we have

$$
\left\langle x+\delta_{i} v-P_{D}\left(x+\delta_{i} v\right), n_{i}\right\rangle \geq 0, \text { for all } i \in N,
$$


which implies

$$
\liminf _{i \in N} \frac{1}{\delta_{i}}\left\langle x-P_{D}\left(x+\delta_{i} v\right), n_{i}\right\rangle \geq \liminf _{i \in N} \frac{1}{\delta_{i}}\left\langle-\delta_{i} v, n_{i}\right\rangle=-\left\langle v, n^{*}\right\rangle>0,
$$

which is impossible since $n^{*} \in \bar{S}^{\sigma} \cap N_{D}(x)$ and $P_{D}\left(x+\delta_{i} v\right) \in D$ for all $i \in N$.

Therefore, there exists $\delta_{*}>0$ such that $x+\delta_{*} v \in D$, which implies (using also the convexity of $D$ ) that

$$
\Pi_{D}(x ; v)=v .
$$

But we know that

$$
\Pi_{D}(x ; v)=v-\left\langle v, n^{*}\right\rangle n^{*} .
$$

From (13) and (14) we obtain that $\left\langle v, n^{*}\right\rangle=0$, which is in a contradiction with our assumption that $\left\langle v, n^{*}\right\rangle<0$. Therefore, in (11) we have $n^{*} \neq 0$ and $\left\langle v, n^{*}\right\rangle \geq 0$.

Now we prove that (11) implies that $n^{*}$ is a maximizer in $\sup _{n \in \bar{S}^{\sigma} \cap N_{D}(x)}\langle v, n\rangle$.

Indeed, since $\left\langle n, n^{*}\right\rangle \leq 1$ for all $n \in \bar{S}^{\sigma} \cap N_{D}(x)$, we have

$$
-\left\langle v, n^{*}\right\rangle \leq-\left\langle v, n^{*}\right\rangle\left\langle n, n^{*}\right\rangle
$$

and also

$$
\langle v, n\rangle-\left\langle v, n^{*}\right\rangle \leq\langle v, n\rangle-\left\langle v, n^{*}\right\rangle\left\langle n, n^{*}\right\rangle
$$

From (15) we deduce

$$
\sup _{n \in \bar{S}^{\sigma} \cap N_{D}(x)}\left[\langle v, n\rangle-\left\langle v, n^{*}\right\rangle\right] \leq \sup _{n \in \bar{S}^{\sigma} \cap N_{D}(x)}\left[\langle v, n\rangle-\left\langle v, n^{*}\right\rangle\left\langle n, n^{*}\right\rangle\right] \leq 0,
$$

which implies

$$
0 \leq\left[\sup _{n \in \bar{S}^{\sigma} \cap N_{D}}\langle v, n\rangle\right]-\left\langle v, n^{*}\right\rangle \leq 0,
$$

that is,

$$
\sup _{n \in \bar{S}^{\sigma} \cap N_{D}(x)}\langle v, n\rangle=\left\langle v, n^{*}\right\rangle,
$$

which means that $n^{*}$ is a maximizer in $\sup _{n \in \bar{S}^{\sigma} \cap N_{D}(x)}\langle v, n\rangle$.

The proof of conclusion (3) will be complete if we show that the maximizer $n^{*}$ is unique. 
Indeed, if we suppose that there exist two maximizers $n_{1}$ and $n_{2}$, then we have

$$
\left\langle v, n_{1}\right\rangle>0 \text { and }\left\langle v, n_{2}\right\rangle>0 .
$$

Since $N_{D}(x)$ is a convex cone, we have that

$$
\frac{n_{1}+n_{2}}{\left\|n_{1}+n_{2}\right\|} \in \bar{S}^{\sigma} \cap N_{D}(x) .
$$

We cannot have $n_{1}=-n_{2}$, since if $n_{1}=-n_{2}$, then $n_{1}+n_{2}=0$, which implies $\left\langle v, n_{1}\right\rangle+\left\langle v, n_{2}\right\rangle=0$, which is impossible considering (16). We have

$$
\left|\left\langle n_{1}, n_{2}\right\rangle\right| \leq 1
$$

or

$$
-1 \leq\left\langle n_{1}, n_{2}\right\rangle \leq 1
$$

but $\left\langle n_{1}, n_{2}\right\rangle \neq 1$. Indeed, if $\left\langle n_{1}, n_{2}\right\rangle=1$ then there exists $\left.\alpha \in\right] 0,1[$ such that $n_{1}=\alpha n_{2}$ and therefore $\left\langle n_{1}, v\right\rangle=\left\langle\alpha n_{2}, v\right\rangle=\alpha\left\langle n_{2}, v\right\rangle\left\langle\left\langle n_{2}, v\right\rangle\right.$ which is impossible since $n_{1}, n_{2}$ are maximizers.

Hence, we have $-1 \leq\left\langle n_{1}, n_{2}\right\rangle<1$, which implies

$$
-2 \leq 2\left\langle n_{1}, n_{2}\right\rangle<2
$$

or

$$
0 \leq 2+2\left\langle n_{1}, n_{2}\right\rangle<4
$$

Using (17) we deduce

$$
\begin{aligned}
0 \leq\left\|n_{1}+n_{2}\right\|^{2} & =\left\langle n_{1}+n_{2}, n_{1}+n_{2}\right\rangle=\left\|n_{1}\right\|^{2}+\left\|n_{2}\right\|^{2}+2\left\langle n_{1}, n_{2}\right\rangle \\
& \leq 2+2\left\langle n_{1}, n_{2}\right\rangle<4,
\end{aligned}
$$

which implies that

$$
\left\|n_{1}+n_{2}\right\|<2, \quad \text { or } \quad \frac{2}{\left\|n_{1}+n_{2}\right\|}>1 .
$$

Finally we have

$$
\frac{\left\langle v, n_{1}+n_{2}\right\rangle}{\left\|n_{1}+n_{2}\right\|}=\frac{\left\langle v, n_{1}\right\rangle+\left\langle v, n_{2}\right\rangle}{\left\|n_{1}+n_{2}\right\|}=\frac{2\left\langle v, n_{1}\right\rangle}{\left\|n_{1}+n_{2}\right\|}>\left\langle n_{1}, v\right\rangle
$$

which is impossible, because $n_{1}$ is a maximizer. 
Therefore we obtain that

$$
\Pi_{D}(x ; v)=v-\left\langle v, n^{*}\right\rangle n^{*},
$$

where $n^{*}$ is the unique maximizer in $\sup _{n \in \bar{S}^{\sigma} \cap N_{D}(x)}\langle v, n\rangle$ and the proof of conclusion (III) of the theorem is complete.

IV. In this case we suppose $x \in \partial D, \sup _{n \in \bar{S}^{\sigma} \cap N_{D}(x)}\langle v, n\rangle=0$ and $\sup _{n \in \bar{S}^{\sigma} \cap N_{D}(x)}\langle v, n\rangle$ has a maximizer $n^{0} \neq 0$.

As in the proof of conclusion (III), we can suppose that $P_{D}(x+\delta) \in \partial D$ for all $\delta>0$, and we can show that

$$
\Pi_{D}(x ; v)=v-\left\langle v, n^{*}\right\rangle n^{*},
$$

where $n^{*} \in \bar{S}^{\sigma} \cap N_{D}(x)$ and $n^{*}$ is the weak limit of a sequence $\left\{n_{i}\right\}_{i \in N}$, where $n_{i} \in n_{D}\left(P_{D}\left(x+\delta_{i} v\right)\right)$, for all $i \in N$.

We note that in an infinite dimensional Hilbert space, $n^{*}$ (in formula (18)) can be zero.

Moreover, we can also show that

$$
\sup _{n \in \bar{S}^{\sigma} \cap N_{D}(x)}\left[\langle v, n\rangle-\left\langle v, n^{*}\right\rangle\left\langle n^{*}, n\right\rangle\right] \leq 0 .
$$

If $n^{*}=0$ then $\left\langle v, n^{*}\right\rangle=0$ and $n^{*}$ is a maximizer in $\sup _{n \in \bar{S}^{\sigma} \cap N_{D}(x)}\langle v, n\rangle$.

If $n^{*} \neq 0$, then we can show that $\left\langle v, n^{*}\right\rangle \geq 0$ (as in the proof of conclusion (III)) and finally we have that $n^{*}$ is a maximizer in $\sup _{n \in \bar{S}^{\sigma} \cap N_{D}(x)}\langle v, n\rangle$.

Therefore we have that $\left\langle v, n^{*}\right\rangle=0$ and using (18) we deduce that $\Pi_{D}(x ; v)=v$.

V. Finally, we suppose that $x \in \partial D$, and we are in any case, not considered in conclusions (I)-(IV).

We know that for any $\delta>0$, the element $P_{D}(x+\delta v)$ is characterized by the following inequality

$$
\left\langle P_{D}(x+\delta v)-(x+\delta v), P_{D}(x+\delta v)-u\right\rangle \leq 0, \quad \text { for all } u \in D .
$$

From the last inequality we have

$$
\lim _{\delta \rightarrow 0_{+}}\left\langle\frac{P_{D}(x+\delta v)-v}{\delta}-v, P_{D}(x+\delta v)-u\right\rangle \leq 0,
$$


which implies

$$
\left\langle\Pi_{D}(x ; v)-v, x-u\right\rangle \leq 0 .
$$

From (20) we deduce $\left\langle v-\Pi_{D}(x ; v), u-x\right\rangle \leq 0$, which implies $v-\Pi_{D}(x ; v) \in$ $N_{D}(x)$, or $\Pi_{D}(x ; v)=v-\xi$, where $\xi$ is an undetermined element in $N_{D}(x)$ and the proof of the theorem is complete.

\section{Consequences for the study of dynamics of solutions to variational inequalities}

The study of equilibrium, as it is considered in Physics or as it is considered in Economics, is based on variational inequalities or on complementarity problems [7], [10], [12], [13], [18], [19], [22], [25], [31]-[36], [45].

We note that the study of equilibrium based on variational inequalities or on complementarity problems is incomplete, since we obtain only a static information. For many practical problems we need to study the equilibrium from the dynamics point of view also. Now this study is realized with the help of projected dynamical systems associated to variational inequalities.

Let $(H,\langle\cdot, \cdot\rangle)$ be an arbitrary Hilbert space, $D \subset H$ a closed convex set and $f: H \rightarrow H$ a mapping. The set $D$ can be a closed convex cone $\boldsymbol{K} \subset H$.

Consider the following variational inequality

$$
V I(f, D):\left\{\begin{array}{l}
\text { find } x_{*} \in D \text { such that } \\
\left\langle x-x_{*}, f\left(x_{*}\right)\right\rangle \geq 0, \text { for all } x \in D .
\end{array}\right.
$$

When the set $D=\boldsymbol{K}$, where $\boldsymbol{K}$ is a closed convex cone, it is well known [24], [25] that the problem $V I(f, D)$ is equivalent to the following complementarity problem :

$$
C P(f, \boldsymbol{K}):\left\{\begin{array}{l}
\text { find } x_{*} \in \boldsymbol{K} \text { such that } \\
f\left(x_{*}\right) \in \boldsymbol{K}^{*} \text { and } \\
\left\langle x_{*}, f\left(x_{*}\right)\right\rangle \geq 0
\end{array}\right.
$$

Given the problem $V I(f, D)$, we consider the ordinary differential equation

$$
\left\{\begin{array}{l}
\frac{d x(t)}{d t}=\Pi_{D}(x(t) ;-f(x(t))) \\
\text { where } x:[0,+\infty[\rightarrow H .
\end{array}\right.
$$


We say that a function $x:[0,+\infty[\rightarrow D$ is a solution to equation (23), if $x(\cdot)$ is an absolutely continuous function and if

$$
\frac{d x(t)}{d t}=\Pi_{D}(x(t) ;-f(x(t)))
$$

for almost all $t \in 0,+\infty[$.

The projected dynamical system defined by the mapping $f$ on the set $D$ (denoted by $P D S(f, D)$, is defined as the mapping $\Phi: D \times \boldsymbol{R} \rightarrow D$ where $\Phi(x, t)=\Phi_{x}(t)$ solves the following initial value problem associated to equation (23), that is :

$$
\left\{\begin{array}{l}
\frac{d \Phi_{x}(t)}{d t}=\Pi_{D}\left(\Phi_{x}(t) ;-f\left(\Phi_{x}(t)\right)\right) \quad \text { for almost all } t . \\
\Phi_{x}(0)=x_{0} \in D
\end{array}\right.
$$

In [36] the $P D S(f, D)$ is studied only in the Euclidean space. In this paper we consider the notion of projected dynamical system in an arbitrary Hilbert space, as it was considered in [26] and also in [3] and [4].

The importance of the notion of projected dynamical system for the study of variational inequalities is related to the following result.

Proposition 6. An element $x_{*} \in D$ is a stationary (equilibrium) point of the projected dynamical system $P D S(f, D)$, i.e. $\Pi_{D}\left(x_{*} ;-f\left(x_{*}\right)\right)=0$, if and only if, $x_{*}$ is a solution of the variational inequality $V I(f, D)$.

Proof. Proposition 6 is a consequence of Theorem 5.

The stability of stationary points of the system $P D S(f, D)$ are studied in [36] only in the Euclidean space using Dupuis' Lemma (Theorem 4).

In the infinite dimensional case, because the conclusions of Theorem 5, we must study the existence of solutions of the problem (24) and the stability of stationary points of the system $P D S(f, D)$ using the following differential inclusion (known also as the "evolution differential inclusion) :

$$
\frac{d x}{d t} \in-f(x)-N_{D}(x),
$$

and considering its "viable solutions".

Generally, if $T$ is a positive real number or $T=+\infty$ we say that a function $x(\cdot):[0, T[\rightarrow H$ is a solution of differential inclusion (25) if $x$ satisfies a continuity property (as for example, absolute continuity, or Lipschitz continuity) and

$$
\frac{d x(t)}{d t} \in-f(x(t))-N_{D}(x(t))
$$

for almost every $t \in[0, T[$. Moreover, if for any $t \in[0, T]$ we have that $x(t) \in D$, we say that $x(t)$ is a viable solution. 
It is known that any solution of equation (23) is a viable solution of the differential inclusion (25), and conversely (see [1] or [3]).

We have the following result.

Proposition 7. An element $x_{*} \in D$ is a solution of the variational inequality $V I(f, D)$ if and only if $x_{*}$ is a stationary (equilibrium) point of the differential inclusion (25), that is $0 \in-f\left(x_{*}\right)-N_{D}\left(x_{*}\right)$.

Proof. The proposition is a consequence of Theorem 5.

Some existence theorems for the differential inclusion (25) in the Euclidean space are given in [5], [20], [21].

The study of existence theorems for the initial value problem (24) in infinite Hilbert spaces is not an easy task, since the operator $\Pi_{D}$ can have discontinuities. However, recently the second author of this paper presented in [3], a nice existence theorem for viable solutions for the differential inclusion (25) in a general Hilbert space. In conclusion, there exists now an existence theorem for the initial value problem (24) in infinite dimensional Hilbert spaces. This existence theorem is the following :

Theorem 8. Let $(H,\langle\cdot, \cdot\rangle)$ be a Hilbert space, $D \subset H$ a non-empty closed convex subset and $f: H \rightarrow H$ a continuous mapping.

Suppose that $f$ satisfies the Lipschitz condition with the Lipschitz constant $b>0$.

Let $x_{0} \in D$ and $L>0$ a real number. Then the initial value problem

$$
\left\{\begin{array}{l}
\frac{d x}{d t} \in-f(x)-N_{D}(x) \\
x(0)=x_{0} .
\end{array}\right.
$$

has a viable solution defined on the interval $\left[0, L_{0}\right]$, where $L_{0}=$ $\frac{L}{\left\|F\left(x_{0}\right)\right\|+b L}$.

Consequently the initial value problem

$$
\left\{\begin{array}{l}
\frac{d x}{d t}=\Pi_{D}(x ;-f(x)) \\
x(0)=x_{0} .
\end{array}\right.
$$

has a solution defined on the interval $\left[0, L_{0}\right]$. Moreover, this solution can be extended to the interval $[0,+\infty[$.

Proof. The proof of this theorem is long and is based on several technical intermediate results. The reader can find this proof in [3] or in [4]. The proof of the extension of this solution is in [4].

Remark. We note that Theorem 8 may replace the existence theorem given in [36], which is given by a long proof based on stochastic processes. 
We note that in the infinite dimensional case, we can have for the differential inclusion (25) solutions in the classical sense or weak solutions, that is, solutions using the derivative with respect to the weak topology. We note that A. Szep remarked this fact in 1971 for ordinary differential equations in reflexive Banach spaces [41].

Now, we will consider this fact for projected dynamical systems in Hilbert spaces.

Definition 1 ([41]). We say that a function $x(\cdot):[0, T[\rightarrow H$ is differentiable at $t_{0} \in[0, T[$, with respect to the weak topology, if and only if, there exists an element $\left[x^{\prime}\left(t_{0}\right)\right]_{w} \in H$ such that

$$
\lim _{t \rightarrow t_{0}} \frac{1}{t-t_{0}}\left\langle\varphi, x(t)-x\left(t_{0}\right)\right\rangle=\left\langle\varphi,\left[x^{\prime}\left(t_{0}\right)\right]_{w}\right\rangle, \text { for any } \varphi \in H
$$

We denote the weak derivative of $x$ at an arbitrary point $t$ also by $\left[\frac{d x(t)}{d t}\right]_{w}$

Definition 2. We say that a function $x(\cdot):[0, T[\rightarrow H$ is a weak viable solution to the differential inclusion (25) if

$$
\left\{\begin{array}{l}
{\left[\frac{d x(t)}{d t}\right]_{w} \in-f(x)-N_{D}(x(t)), \text { for almost every } t \geq t_{0},} \\
x\left(t_{0}\right)=x_{0}, \text { and } x(t) \in D \text { for any } t \geq t_{0} .
\end{array}\right.
$$

Remark. Any solution to the differential inclusion (25), given by Theorem 8 is in particular a weak solution in the sense of Definition 2.

Let $(H,\langle\cdot, \cdot\rangle)$ be a Hilbert space, $D \subset H$ a non-empty, closed convex subset and $f: D \rightarrow H$ a function, weak-to-weak continuous. Then the differential inclusion (25) has a weak solution.

Indeed, $0 \in N_{D}(x)$, for any $x \in D$, so the set valued mapping

$$
\varphi(x):=-f(x)-N_{D}(x)
$$

has a weak-to-weak continuous selection given by $x \rightarrow f(x)$. Since the initial value problem

$$
\left\{\begin{array}{l}
\frac{d x(t)}{d t}=f(x(t)) \\
x(0)=x_{0}, x_{0} \in D,
\end{array}\right.
$$

has a weak solution (see [41]), then the differential inclusion (25) has a weak solution. In this general situation we do not know if this weak solution is a viable solution. The existence of weak viable solutions for the differential inclusion (25), when this inclusion does not have a classical solution is an open problem and we will consider it in a future paper. 
The authors of the book [36] proved in the Euclidean space several stability results for the stationary points of the differential equation (23) supposing that $f$ is monotone or locally monotone mapping. Other stability results are given in [10], [12], [33]-[36], [45].

We note that, some of these results are valid in the infinite dimensional Hilbert spaces when the equation (23) has classical solutions, (i.e. the derivative is considered with respect to the norm topology). Moreover, these results are valid in infinite Hilbert spaces replacing the monotonicity by pseudo-monotonicity [3].

Now, we consider the stability of stationary points, with respect to weak viable solutions, for the differential inclusion (25) in an arbitrary Hilbert space, supposing that weak viable solutions exist.

Definition 3. We say that $x_{0} \in D$ is a strict stationary point of the differential inclusion (25) if there exists $n_{0} \in N_{D}\left(x_{0}\right)$ such that

(1) $-f\left(x_{0}\right)-n_{0}=0$ and

(2) $\left\langle y-x_{0}, n_{0}\right\rangle<0$, for any $y \in D, y \neq x_{0}$.

Remark. From Theorem 5 we have that if intD is non-empty, then a strict stationary point can not be in intD.

We recall that a mapping $f: H \rightarrow H$ is called pseudo-monotone on the set $D$, if and only if, for any $x, y \in D$, we have that $\langle y-x, f(x)\rangle \geq 0$ implies that

$$
\langle y-x, f(y)\rangle \geq 0 .
$$

Also, we say that a mapping $f: H \rightarrow H$ is quasi-monotone on $D$ if and only if, for every pair of distinct points $x, y \in D$, we have that $\langle y-x, f(x)\rangle>0$ implies that

$$
\langle y-x, f(y)\rangle \geq 0 .
$$

If the pseudo-monotonicity (resp. quasi-monotonicity) condition is satisfied in a neighborhood of any point of $D$, then we say that $f$ is locally pseudomonotone (resp. locally quasi-monotone).

It is known [28] that any monotone mapping is pseudo-monotone and any pseudo-monotone is quasi-monotone but the converse is not true.

The reader can find many results about monotone mappings in [6], [8], [9], [17],[19], [27]-[29], [37].

\section{Definition 4.}

(i) We say that a stationary point $x_{*} \in D$ of the differential inclusion (25) is a monotone attractor for viable solutions, if there exists $\delta>0$ such that for any $x \in B\left(x_{*}, \delta\right)=\left\{x \in D \mid\left\|x-x_{*}\right\|<\delta\right\}$ the function $d(x ; t)=\left\|x(t)-x_{*}\right\|$ is non-increasing as function of $t$, for almost every $t \geq 0$, where $x(t)$ is viable solution of the differential inclusion (25) starting at the point $x$. 
(ii) If in definition (i) we replace $x(t)$ by a weak viable solution, then we say that $x_{*}$ is a monotone attractor for weak viable solutions.

If in definition (i) we replace $x(t)$ by any viable solution (respective by any weak viable solution) starting to any $x \in D$, then we say that $x_{*}$ is a global monotone attractor for viable solutions (resp. for weak viable solutions).

The following theorem is necessary for our stability result, with respect to weak viable solutions.

Theorem 9. Let $\langle H,\langle\cdot, \cdot\rangle\rangle$ be a Hilbert space, $x: \boldsymbol{R} \rightarrow H$ a function, $t_{0} \in \boldsymbol{R}$ and $\left[\frac{d x}{d t}\left(t_{0}\right)\right]_{w}$ an element of $H$ such that :

$$
\lim _{t \rightarrow t_{0}} \frac{1}{t-t_{0}}\left\langle\xi, x(t)-x\left(t_{0}\right)\right\rangle=\left\langle\xi,\left[\frac{d x}{d t}\left(t_{0}\right)\right]_{w}\right\rangle, \text { for any } \xi \in H .
$$

Then we have

$$
\lim _{t \rightarrow t_{0}} \frac{1}{t-t_{0}}\left[\frac{\|x(t)\|^{2}}{2}-\frac{\left\|x\left(t_{0}\right)\right\|^{2}}{2}\right]=\left\langle x\left(t_{0}\right),\left[\frac{d x}{d t}\left(t_{0}\right)\right]_{w}\right\rangle .
$$

Proof. Let $\left\{t_{n}\right\}_{n \in N}$ be a sequence in $\boldsymbol{R}$ convergent to $t_{0}$. For any $n \in N$ we denote by $u_{n}: H \rightarrow H$ the mapping defined by

$$
\xi \rightarrow \frac{1}{t_{n}-t_{0}}\left\langle\xi, x\left(t_{n}\right)-x\left(t_{0}\right)\right\rangle \text {. }
$$

For any $n \in N, u_{n}$ is a continuous linear mapping. Hence, $\left\{u_{n}\right\}_{n \in N}$ is a sequence of continuous linear functionals on $H$ such that

$$
\lim _{n \rightarrow \infty} u_{n}(\xi)=\left\langle\xi,\left[\frac{d x}{d t}\left(t_{0}\right)\right]_{w}\right\rangle \text {, for any } \xi \in H .
$$

By the Banach-Steinhaus Theorem (See [43]), we have that

$$
\alpha:=\sup _{n \in N}\left\|u_{n}\right\|<\infty \text {. }
$$

Then, we have

$$
\left\|x\left(t_{n}\right)-x\left(t_{0}\right)\right\| \leq \alpha\left|t_{n}-t_{0}\right|, \text { for any } n \in N .
$$

From the following equality

$$
\begin{aligned}
\left\|x\left(t_{n}\right)\right\|^{2}-\left\|x\left(t_{0}\right)\right\|^{2} & =\langle x(t), x(t)\rangle-\left\langle x\left(t_{0}\right), x\left(t_{0}\right)\right\rangle \\
& =2\left\langle x\left(t_{0}\right), x(t)-x\left(t_{0}\right)\right\rangle+\left\langle x(t)-x\left(t_{0}\right), x(t)-x\left(t_{0}\right)\right\rangle,
\end{aligned}
$$

which is valid for any $t \in \boldsymbol{R}$, we have

$$
\begin{aligned}
& \left|\frac{1}{2\left(t_{n}-t_{0}\right)}\left(\left\|x\left(t_{n}\right)\right\|^{2}-\left\|x\left(t_{0}\right)\right\|^{2}\right)-\left\langle x\left(t_{0}\right),\left[\frac{d x}{d t}\left(t_{0}\right)\right]_{w}\right\rangle\right| \\
& \quad \leq\left|\frac{1}{t_{n}-t_{0}}\left\langle x\left(t_{0}\right), x\left(t_{n}\right)-x\left(t_{0}\right)\right\rangle-\left\langle x\left(t_{0}\right),\left[\frac{d x}{d t}\left(t_{0}\right)\right]_{w}\right\rangle\right|+\frac{\alpha^{2}}{2}\left|t_{n}-t_{0}\right|,
\end{aligned}
$$


for any $n \in N$ and hence

$$
\lim _{n \rightarrow \infty}\left|\frac{1}{2\left(t_{n}-t_{0}\right)}\left(\left\|x\left(t_{n}\right)\right\|^{2}-\left\|x\left(t_{0}\right)\right\|^{2}\right)-\left\langle x\left(t_{0}\right),\left[\frac{d x}{d t}\left(t_{0}\right)\right]_{w}\right\rangle\right|=0 .
$$

Therefore, the proof is complete.

Now, we give a stability theorem for the following initial-value problem :

$$
\left\{\begin{array}{l}
\text { find a viable solution for } \\
\text { the differential inclusion } \\
{\left[\frac{d x}{d t}\right]_{w} \in-f(x)-N_{D}(x)} \\
x(0)=x_{0}, x_{0} \in D
\end{array}\right.
$$

For our next stability theorem we suppose that problem (26) has a solution for any $x_{0} \in D$, for example when the assumptions of Theorem 8 are satisfied.

Theorem 10. If $x_{*} \in D$ is a stationary point (respectively a strict stationary point) of the differential inclusion (26) and $f$ is pseudo-monotone (respectively quasi-monotone), then $x_{*}$ is global monotone attractor for viable solutions of (26) (i.e., for viable weak solution of (25).

Proof. First, we show that if $x(t)$ is a viable solution to the differential inclusion (26), starting at a point $x_{0} \in D$, then we have

$$
\frac{d}{d t}\left(\frac{1}{2}\left\|x(t)-x_{*}\right\|^{2}\right) \leq 0, \text { for almost every } t \geq 0 .
$$

Indeed, since $x(t)$ is a solution to the differential inclusion (26), then we have

$$
\left[\frac{d x(t)}{d t}\right]_{w} \in-f(x(t))-N_{D}(x(t)), \text { for almost every } t \geq 0,
$$

which implies that, there exists $n \in N_{D}(x(t))$ such that

$$
\left[\frac{d x(t)}{d t}\right]_{w}=-f(x(t))-n, \text { for almost every } t \geq 0 .
$$

Applying Theorem 9 we have

$$
\begin{aligned}
\frac{d}{d t}\left(\frac{1}{2}\left\|x(t)-x_{*}\right\|^{2}\right) & =\left\langle x(t)-x_{*},\left[\frac{d x}{d t}\right]_{w}\right\rangle \\
& =\left\langle x(t)-x_{*},-f(x(t))-n\right\rangle \\
& =\left\langle x(t)-x_{*},-f(x(t))\right\rangle+\left\langle x(t)-x^{*},-n\right\rangle,
\end{aligned}
$$

for almost every $t \geq 0$. From the definition of $N_{D}(x(t))$ we have

$$
\left\langle x(t) x_{*},-n\right\rangle=\left\langle x_{*}-x(t), n\right\rangle \leq 0 .
$$


Since $x_{*}$ is a stationary point of the differential inclusion (26), we have

$$
0 \in-f\left(x_{*}\right)-N_{D}\left(x_{*}\right),
$$

i.e., there exists, $n_{*} \in N_{D}\left(x_{*}\right)$ such that $-f\left(x_{*}\right)=n_{*}$, which implies

$$
\left\langle x(t)-x_{*},-f\left(x_{*}\right)\right\rangle=\left\langle x(t)-x_{*}, n_{*}\right\rangle \leq 0,
$$

that is, $\left\langle x(t)-x_{*}, f\left(x_{*}\right)\right\rangle \geq 0$ and by pseudomonotonicity we have $\left\langle x(t)-x_{*}, f(x(t))\right\rangle \geq 0$, that is we have

$$
\left\langle x(t)-x_{*},-f(x(t))\right\rangle \leq 0 .
$$

Similarly, if $x_{*}$ is a strict stationary point of the differential inclusion (26) and $f$ is quasi-monotone we have,

$$
\left\langle x(t)-x_{*},-f\left(x_{*}\right)\right\rangle=\left\langle x(t)-x_{*}, n_{*}\right\rangle<0 \text {, for any } t \text { such that } x(t) \neq x_{*}
$$

and by quasi-monotonicity we have again that (29) is true.

Therefore, from (27), (28) and (29) we obtain that

$$
\frac{d}{d t}\left(\frac{1}{2}\left\|x(t)-x_{*}\right\|^{2}\right) \leq 0 \text {, for almost every } t \geq 0 .
$$

From (30) we deduce immediately that, the function $d(x ; t)=\left\|x(t)-x_{*}\right\|$ is non-increasing as function of $t$, for almost every $t \geq 0$.

Therefore, we deduce that $x_{*}$ is a global monotone attractor for viable weak solutions.

Remark. If $f$ is locally pseudo-monotone (respectively, locally quasimonotone), then using a similar computation, but locally, we deduce that $x_{*}$ is a monotone attractor for viable weak solutions.

Now, we indicate another way, which can be exploited to obtain new existence theorems for projected dynamical systems in arbitrary Hilbert spaces. This way is based on the Nagumo's Theorem and the selections for set-valued mappings. Before to explain this idea, we recall the Nagumo's Theorem.

Theorem 11 ([Nagumo]). Let $D$ be a closed subset of an arbitrary Hilbert space $(H,\langle\cdot, \cdot\rangle)$ and $\psi: D \rightarrow H$ a continuous function. If for any $x \in D$, we have that $\psi(x) \in T_{D}(x)$, then for any $x_{0} \in D$, there exists a real number $T_{0}>0$ such that the differential equation

$$
\left\{\begin{array}{l}
\frac{d x(t)}{d t}=\psi(x(t)) \\
x(0)=x_{0} .
\end{array}\right.
$$

has a viable solution on $\left[0, T_{0}\right]$.

Proof. See ([1], Theorem 2, p. 180).

Theorem 12 ([Moreau]). If $\boldsymbol{K} \subset H$ is a closed convex cone and $x, y, z \in H$, then the following statements are equivalent :

(1) $z=x+y, x \in \boldsymbol{K}, y \in \boldsymbol{K}^{0}$ and $\langle x, y\rangle=0$, 
(2) $x=P_{k}(z)$ and $y=P_{\boldsymbol{K}^{0}}(z)$.

Proof. See ([22], Theorem 2.23, p. 18).

If $A \subset H$ is a non-empty subset and $\varepsilon>0$, we denote by $B(A, \varepsilon)$ the set $\{x \in H \mid d(x, A)<\varepsilon\}$, where $d(x, A)=\inf _{y \in A} d(x, y)$.

Let $X \subset H$ be an arbitrary non-empty set. We recall the following definitions :

(a) We say that a set-valued mapping $\Gamma: X \rightarrow H$ is almost lower semicontinuous (a.l.s.c.) at $x_{0} \in X$ if for each $\varepsilon>0$ there exists a neighborhood $V\left(x_{0}\right)$ such that

$$
\bigcap_{x \in V\left(x_{0}\right)} B(\Gamma(x), \varepsilon) \neq \phi .
$$

(b) Supposing $r \in] 0,1]$, we say that a set-valued mapping $\Gamma: X \rightarrow H$ is $r$-lower semicontinuous (r-l.s.c.) at $x_{0} \in X$ if :

(i) $\Gamma$ is a.l.s.c. at $x_{0}$ and

(ii) for every $\varepsilon>0$ and every neighborhood $V\left(x_{0}\right)$ of $x_{0}$, if $y_{0} \in$ $\bigcap_{x \in V\left(x_{0}\right)} B(\Gamma(x), \varepsilon)$, then there exists a neighborhood $V\left(x_{1}\right)$ of every $x_{1} \in V\left(x_{0}\right)$ such that $y_{0} \in B\left(\bigcap_{x \in V\left(x_{1}\right)} B(\Gamma(x), r \varepsilon), \varepsilon\right)$

If $\Gamma$ is r-l.s.c. at each point of $X$, we say that $\Gamma$ is $r$-lower semicontinuous.

H.X. Li and F.L. Huang proved in [30] the following result.

Theorem 13. Let $X$, be a paracompact space and $Y$ a Banach space. Then every $r$-lower semicontinuous set-valued mapping $\Gamma: X \rightarrow Y$ with closed convex values admits a continuous selection where $r \in] 0,1[$.

Theorem 14. Let $(H,\langle\cdot, \cdot\rangle)$ be a Hilbert space, $D \subset H$ a closed convex set and $f: D \rightarrow H$ a continuous function. Then the set-valued mapping

$$
\Gamma(x)=\left\{-f(x)-\xi \mid \xi \in N_{D}(x),\|\xi\| \leq\|f(x)\|\right\} \cap T_{D}(x)
$$

is such that for any $x \in D, \Gamma(x)$ is non-empty.

If $\Gamma$ is r-lower semicontinuous, then for any $x_{0} \in D$ there exists $T_{0}>0$ such that the differential inclusion (25) has a viable solution on $\left[0, T_{0}\right]$ starting at the point $x_{0}$.

Proof. First, we will show that for any $x \in D, \Gamma(x)$ is non-empty.

Indeed, let $x \in D$, be an arbitrary element. Applying Moreau's Theorem we have

$$
-f(x)=P_{T_{D}(x)}(-f(x))+P_{N_{D}(x)}(-f(x)) .
$$

From (31) we deduce that

$$
P_{N_{D}(x)}(-f(x))=-f(x)-P_{T_{D}(x)}(-f(x))
$$


which implies

$$
\begin{aligned}
\left\|P_{N_{D}(x)}(-f(x))\right\| & =\left\|-f(x)-P_{T_{D}(x)}(-f(x))\right\| \\
& =\inf _{y \in T_{D}(x)}\|-f(x)-y\| \\
& \leq\|f(x)\| .
\end{aligned}
$$

Because $\left\|P_{N_{D}(x)}(-f(x))\right\| \leq\|f(x)\|$ and $-f(x)-P_{N_{D}(x)}(-f(x))=$ $P_{T_{D}(x)}(-f(x)) \in T_{D}(x)$, we deduce that $-f(x)-P_{N_{D}(x)}(-f(x)) \in \Gamma(x)$, that is $\Gamma(x)$ is non-empty.

Using the fact that $N_{D}(x)$ and $T_{D}(x)$ are closed and the norm $\|\cdot\|$ is continuous, we have that for any $x \in D, \Gamma(x)$ is closed. Also we can show that, the set

$$
\left\{-f(x)-\xi \mid \xi \in N_{D}(x),\|\xi\| \leq\|f(x)\|\right\}
$$

is convex and because $T_{D}(x)$ is convex too, we obtain that for any $x \in D$, $\Gamma(x)$ is convex. In conclusion, for any $x \in D$, the set $\Gamma(x)$ is a closed convex set.

Since, by assumption $\Gamma$ is $r$-lower semicontinuous on $D$, we obtain by Theorem 12 that $\Gamma$ has a continuous selection. Let $\psi$ be this continuous selection. By construction the function $\psi$ satisfies the assumptions of Nagumo's Theorem and the conclusion of theorem follows, since a viable solution of the differential equation $\frac{d x}{d t}=\psi(x)$ is a viable solution of the differential inclusion (25).

\section{Comments}

In this paper we presented several results concerning the development of the theory of projected dynamical systems in arbitrary Hilbert spaces. This theory started recently in our papers: [26], [3], [4].

With projected dynamical systems we can study variational inequalities and complementarity problems in arbitrary Hilbert spaces from the point of view of dynamical systems, that is, the behavior of their solutions with respect to the time or with respect to another parameter, when some constraints must be respected.

To develop this theory, several facts must be considered. For example:

(1) the existence of weak viable solutions for the differential inclusion (25) in more general situation,

(2) it is interesting to find new existence theorems for classical solutions, using for example the methods presented in the book [42].

(3) it is interesting to study the possibility to obtain new existence theorems by the fixed point theory,

(4) it is interesting to find new stability theorems and applications to practical problems. 
Such kind of problems may be considered in future papers.

Acknowledgements. The authors are grateful to the anonymous referee for his valuable comments and for his list of missprints

\section{References}

[1] J. P. Aubin and A. Celina, Differential Inclusions, Springer-Verlag, Berlin, 1984.

[2] C. Baiocchi and A. Capello, Variational and Quasi-Variational Inequalities. Applications to Free Boundary Problems, John Wiley and Sons, 1984.

[3] M. G. Cojocaru, Projected Dynamical Systems on Hilbert Spaces, Ph.D. Thesis, Queen's University, 2002.

[4] M. G. Cojocaru and L. B. Jonker, Existence of solutions to projected differential equations in Hilbert spaces, Proc Amer. Math. Soc., to appear.

[5] B. Cornet, Existence of slow solutions for a class of differential inclusion, J. Math. Anal. Appl., 96 (1983), 130-147.

[6] R. W. Cottle and J. C. Yao, Pseudo-monotone complementarity problems in Hilbert spaces, J. Opt. Theory Appl., 75 (2) (1992), 281295.

[7] R. W. Cottle, J. S. Pang and R. E. Stone, The Linear Complementarity Problem, Academic Press, New York, 1992.

[8] J. P. Crouzeix, Pseudo-monotone variational inequality problems: existence of solutions, Math. Programming, 78 (1997), 305-314.

[9] J. Crouzeix and J. A. Ferland, Criteria of differentiable generalized monotone maps, Math. Programming 75 (1996), 399-406.

[10] J. Dong, D. Zhang and A. Nagurney, A projected dynamical system model of general financial equilibrium with stability analysis, Mathematical and Computer Modeling, 24 (1996), 35-44.

[11] P. Dupuis, Large deviations analysis of reflected diffusions and constrained stochastic approximation algorithms in convex set, Stochatics, 21 (1987), 63-96.

[12] P. Dupuis and A. Nagurney, Dynamical systems and variational inequalities, Annals of Operations Research, 44 (1993), 9-42.

[13] M. C. Ferris and J. S. Pang, Engineering and economic applications of complementarity problems, Discussion Paper in Economics, Department of Economics, Univ. Colorado-Boulder, May, 1995. 
[14] A. N. Godunov, Peano's theorem in Banach spaces, Funktsional'nyi Analiz i Ego Prilozheniya, 9 (1) (1975), 59-60.

[15] L. Gorniewicz, Topological Fixed Point Theory of Multivalued Mappings, Kluwer Academic Publishers, 1999.

[16] M. S. Gowda, Affine pseudo-monotone mappings and the linear complementarity problems, SIAM J. on Matrix Anal. Appl., 11 (1990), 373-380.

[17] N. Hadjisavvas and S. Schaible, Quasimonotone variational inequalities in Banach spaces, J. Opt. Theory Appl., 90 (1) (1996), 95-111.

[18] N. Hadjisavvas and S. Schaible, From scalar to vector equilibrium problems in the quasimonotone case, J. Opt. Theory Appl., 96 (2) (1998), 297-309.

[19] N. Hadjisavvas and S. Schaible, Quasimonotonicity and pseudomonotonicity in variational inequalities and equilibrium problems, In : Generalized Convexity and Monotonicity : Recent Developments, Eds. J. P. Crouzeix, J. E. Martinez-Legas and M. Volle, Kluwer Academic Publishers, to appear.

[20] C. Henry, Differential equations with discontinuous right hand sides for planning procedures, J. Econom. Theory, 4 (1972), 545-551.

[21] C. Henry, An existence theorem for a class of differential equations with multivalued right hand sides, J. Math Anal. Appl. 41 (1973), 179-186.

[22] D. H. Hyers, G. Isac and Th. M. Rassias, Topics in Nonlinear Analysis and Applications, World Scientific, Singapore, New Jersey, London, Hong Kong, 1997.

[23] G. Isac, Nonlinear complementarity problem and Galerkin method, J. Math. Anal. Appl., 108 (2) (1985), 563-574.

[24] G. Isac, Complementarity Problems, Lecture Notes in Math., SpringerVerlag, Nr. 1528, 1992.

[25] G. Isac, Topological Methods in Complementarity Theory, Kluwer Academic Publishers, 2000.

[26] G. Isac and M. G. Cojocaru, Variational inequalities, complementarity problems and pseudo-monotonicity. Dynamical aspects, Seminar on Fixed Point Theory, Cluj-Napoca (Proceedings of the International Conference on Nonlinear Operators, Differential Equations and Applications, September 2001, Babes, Bolyai University, Cluj-Napoca, Romania).

[27] S. Karamardian, Complementarity Problems over cones with monotone and pseudo-monotone maps, J. Opt. Theory Appl., 18 (1976), 445-454.

[28] S. Karamardian and S. Schaible, Seven kinds of monotone maps, J. Opt. Theory Appl., 66 (1) (1990), 37-46. 
[29] S. Karamardian, S. Schailbe and J. P. Crouzeix, Characterizations of general monotone maps, J. Opt. Theory Appl., 76 (1993), 399-413.

[30] H. X. Li, and F. L. Huang, Continuous selections for multivalued mappings with closed convex images and applications, J. Math. Anal. Appl., 252 (2000), 402-409.

[31] A. Nagurney, Network Economics. A variational Inequality Approach, Kluwer Academic Publishers, 1993.

[32] A. Nagurney, P. Dupuis and D. A. Zhang, A dynamical system approach for network oligopolies and variational inequalities, Annals of Regional Science, 28 (1994), 263-283.

[33] A. Nagurney, T. Takayama and D. A. Zhang, Projected dynamical systems modeling and computation of spatial network equilibria, Networks, 26 (1995), 69-85.

[34] A. Nagurney and D. A. Zhang, On the stability of an adjustment process for spatial price equilibrium modeled as a projected dynamical system, J. Economic Dynamics and Control, 20 (1996), 43-63.

[35] A. Nagurney and D. A. Zhang, Stability analysis of an adjustment process for oligopolistic market equilibrium modeled as a projected dynamical system, Optimization, 36 (1996), 263-285.

[36] A. Nagurney and D. A. Zhang, Projected Dynamical Systems and Variational Inequalities with Applications, Kluwer Academic Publishers, Boston, Massachusetts, 1996.

[37] S. Schaible, Generalized monotonicity, concepts and uses, In: Variational Inequalities Network Equilibrium Problems, (Eds. F. Giannessi and A. Maugeri), Plenum, New York (1995), 289-299.

[38] A. Shapiro, On differentiability of metric projections in $\boldsymbol{R}^{n}, 1$ : Boundary case, Proc. Amer. Math. Soc., 99 (1987), 123-128.

[39] A. Shapiro, Directional differentiability of metric projections onto moving sets at boundary points, J. Math. Anal. Appl., 131 (1998), 392-403.

[40] A. Shapiro, Existence and differentiability of metric projections in Hilbert spaces, SIAM J. Optimization, 4 (1) (1994), 130-141.

[41] A. Szep, Existence theorems for weak solutions of ordinary differential equations in reflexive Banach spaces, Studia Scientiarum Matematicarum Hungarica, 6 (1971), 197-203.

[42] A. Tolstonogov, Differential Inclusions in a Banach Space, Kluwer Academic Publishers, 2000.

[43] P. Wojtaszczyk, Banach spaces for Analysts, Cambridge University Press, New York, 1991. 
[44] E. H. Zarantonello, Projections on convex sets in Hilbert spaces and spectral theory, In : Contributions to Nonlinear Functional Analysis, (Ed. E. H. Zarantonello), Academic Press, New York (1971), 237-424.

[45] D. Zhang and A. Nagurney, On the stability of projected dynamical systems, J. Opt. Theory Appl., 83 (2) (1994), 391-403.

Department of Mathematics

Royal Military College of Canada

P.O. Box 17000 STN Forces

Kingston, Ontario

Canada, K7K 7B4

(Email : isac-g@rmc.ca)

Department of Mathematics and Statistics

Queen's University, Jeffery Hall

Kingston, Ontario

Canada, K7L 3N6

(Email : monica@mats.QueensU.ca) 


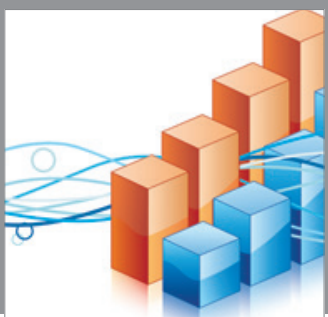

Advances in

Operations Research

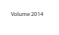

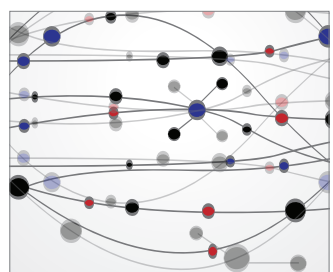

\section{The Scientific} World Journal
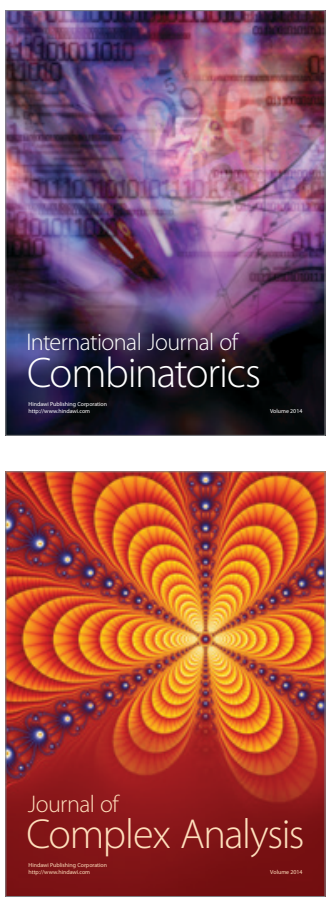

International Journal of

Mathematics and

Mathematical

Sciences
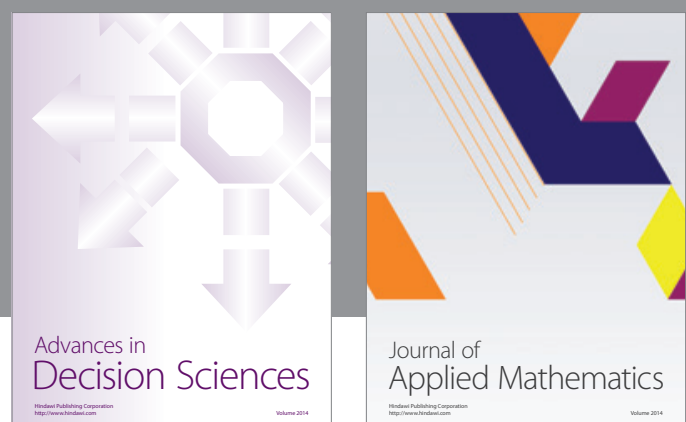

Journal of

Applied Mathematics
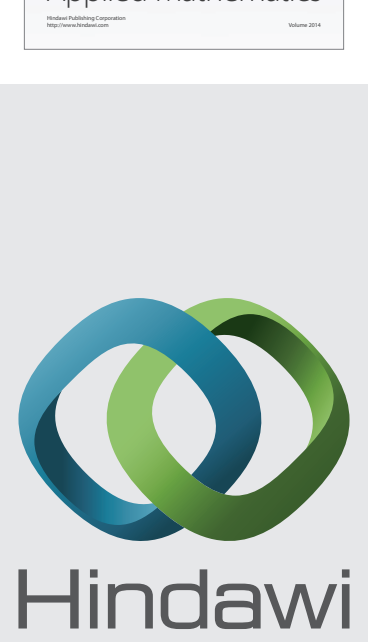

Submit your manuscripts at http://www.hindawi.com
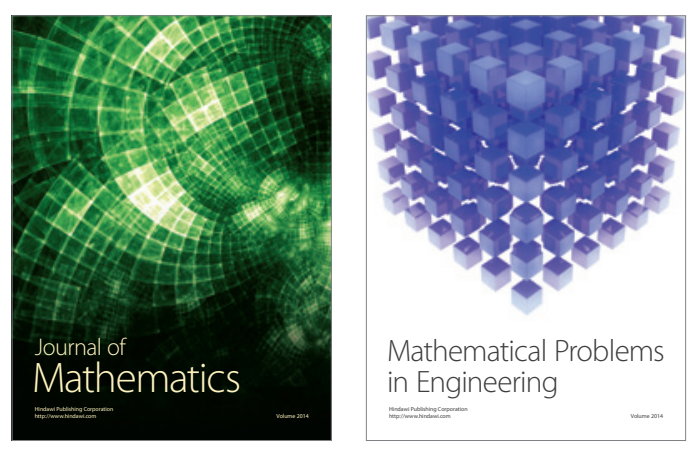

Mathematical Problems in Engineering
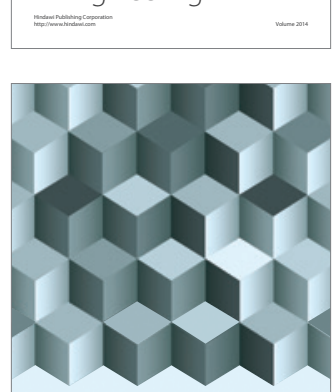

Journal of

Function Spaces
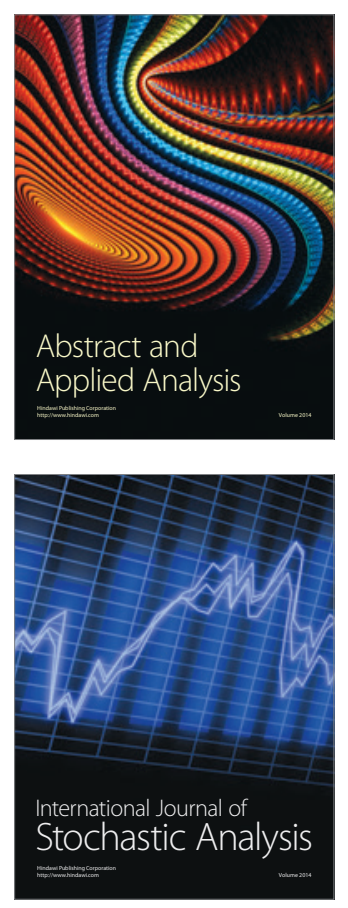

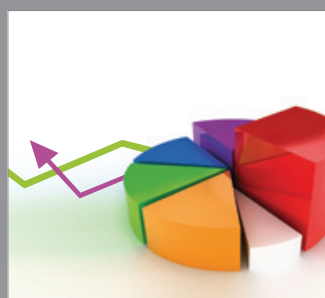

ournal of

Probability and Statistics

Promensencen
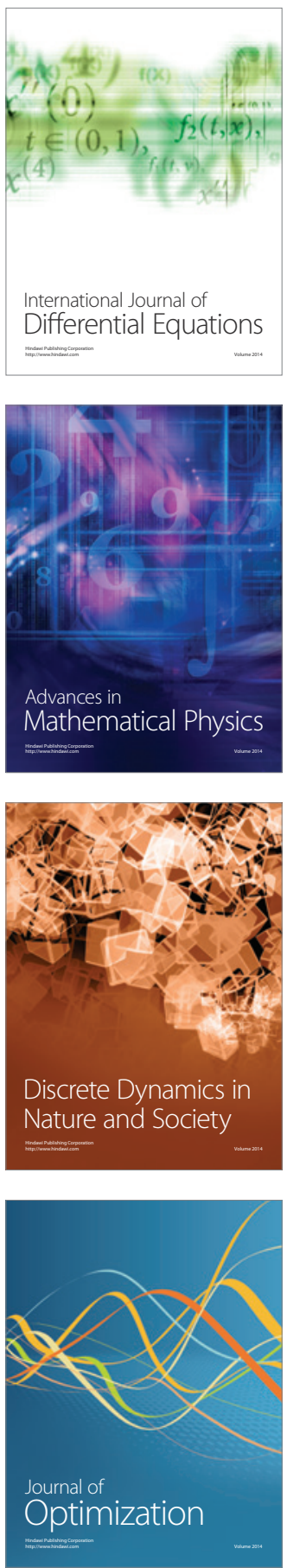